Ana L. Barrenechea $($ ) Carlos C. Peña $(1)$

\title{
Stability of 2nd conjugate Banach algebras
}

Received: 19 September 2017 / Accepted: 28 January 2018 / Published online: 15 February 2018

(c) The Author(s) 2018. This article is an open access publication

\begin{abstract}
In general the stability of normed algebras is a non hereditary property. We shall prove that second conjugate Banach algebras may be non stable even if the underlying Banach algebra is stable. We shall characterize stability of second conjugate Banach algebras. Finally, we shall study kinds of stability induced on an algebra with an stable second conjugate algebra.
\end{abstract}

Mathematics Subject Classification Primary 46H99 - Secondary 47B48

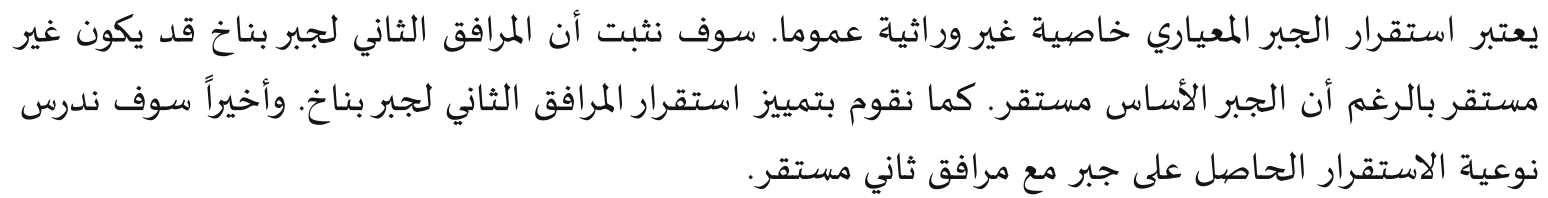

\section{Introduction}

Throughout this article, $A$ will be a complex Banach algebra. Then $A$ is called left (resp., right) stable if the left (resp., right) regular representation $L_{A}$ (resp., anti-representation $R_{A}$ ) of $A$ into $B(A)$ is isometric. $A$ is stable if it is left and right stable. Recently, stable Banach algebras became relevant because of their important role in the theory of almost multiplier maps [1]. It is readily seen that the class of left (resp., right) stable algebras contains the class of Banach algebras endowed with a bounded right (resp., left) approximate identity with bound 1. Likewise, any stable Banach algebra is faithful. However, there are stable Banach algebras without bounded approximate units and there are faithful non stable Banach algebras. Further, subalgebras of stable Banach algebras are not necessarily stable [2].

Let $\chi_{A}$ be the isometric immersion $\chi_{A}: A \hookrightarrow A^{* *}$ of $A$ into its second conjugate space $A^{* *}$. It is known that $A^{* *}$ has two Banach algebra structures with products $\square$ and $\diamond$ that generalize the underlying product of $A[3,4]$. This fact gave raise to the important issue of algebraic (or Arens) regularity, related to deciding

A. L. Barrenechea $\cdot$ C. C. Peña $(\varangle)$

UNCPBA, FCExactas, Dpto. Matemáticas, NUCOMPA, Campus Universitario, Tandil, Pcia. Bs. As., Argentina

E-mail: ccpenia@gmail.com

A. L. Barrenechea

E-mail: analucia.barrenechea@gmail.com 
conditions of coincidence of these products. Given $a^{* *}, b^{* *} \in A^{* *}$ we shall write

$$
\begin{aligned}
& a^{* *} \square b^{* *}=w^{*}-\lim _{i \in I} \lim _{j \in J} \chi_{A}\left(a_{i} b_{j}\right), \\
& a^{* *} \diamond b^{* *}=w^{*}-\lim _{j \in J} \lim _{i \in I} \chi_{A}\left(a_{i} b_{j}\right),
\end{aligned}
$$

where $\left\{a_{i}\right\}_{i \in I}$ and $\left\{b_{j}\right\}_{j \in J}$ are bounded nets in $A$ so that

$$
a^{* *}=w^{*}-\lim _{\in I} \chi_{A}\left(a_{i}\right) \text { and } b^{* *}=w^{*}-\lim _{j \in J} \chi_{A}\left(b_{j}\right)
$$

(cf. [7], 1.4., 46-64). Since its introduction in 1951, this setting constituted an important way for the development of Banach algebra theory. We remark the following two facts: (i) Any Banach algebra $A$ becomes a subalgebra of its second dual Banach algebra $\left(A^{* *}, \square\right)$ (or $(A, \diamond)$ ). (ii) Stability is not an hereditary property. Thus, it is of interest to seek on relationships of stability of a Banach algebra and stability of the corresponding second dual Banach algebras (cf. [2], 4., Open Problem 2).

Our aim is to investigate the relationship between the stability of $A^{* *}$ and the stability of $A$. To this end, in Lemma 2.1 we shall relate the left regular representations of $A$ and $A^{* *}$. In our main result Th. 2.3 we shall see that the underlying algebra $A$ inherits the same kind of stability of its second conjugate Banach-Arens algebras. In Ex. 2.4 we shall exhibit a stable Banach abelian algebra whose second conjugate algebra is not stable. This example gives a negative answer to Problem (2) posed in [2]. As stability is a metric condition, the characterization in Th. 2.5 of stable second conjugate Banach algebras relies on metric facts explored in Lemma 2.2.

\section{Algebraic stability of the 2 nd conjugate algebra}

Lemma 2.1 There is a contractive linear operator $\Gamma: \mathfrak{B}(A)^{* *} \rightarrow \mathfrak{B}\left(A^{* *}\right)$ so that $L_{\square}=\Gamma \circ\left(L_{A}\right)^{* *}$, $R_{\diamond}=\Gamma \circ\left(R_{A}\right)^{* *}$, where $L_{\square}$ and $R_{\diamond}$ denote the left and right regular representation and anti-representation of $A^{* *}$ endowed with the Arens products $\square$ and $\diamond$, respectively.

Proof Let $u: A^{*} \times A^{* *} \rightarrow \mathfrak{B}(A)^{*}$ so that $u\left(a^{*}, a^{* *}\right)(T)=\left\langle T^{*}\left(a^{*}\right), a^{* *}\right\rangle$ if $T \in \mathfrak{B}(A), a^{*} \in A^{*}$ and $a^{* *} \in$ $A^{* *}$. It is plain that $u$ is a well defined norm-one bilinear form and if $n \in \mathfrak{B}(A)^{* *}$ we set $\left\langle a^{*}, \Gamma(n)\left(a^{* *}\right)\right\rangle=$ $\left\langle u\left(a^{*}, a^{* *}\right), n\right\rangle$. It is readily seen that $\Gamma$ becomes a well defined contractive linear operator between $\mathfrak{B}(A)^{* *}$ and $\mathfrak{B}\left(A^{* *}\right)$.

Let $a \in A, a^{*} \in A^{*}$ and $a^{* *}, b^{* *} \in A^{* *}$. Then,

$$
\begin{aligned}
\left\langle a,\left(L_{A}\right)^{*}\left[u\left(a^{*}, b^{* *}\right)\right]\right\rangle & =\left\langle L_{A}(a), u\left(a^{*}, b^{* *}\right)\right\rangle \\
& =\left\langle\left(L_{A}\right)^{*}\left(a^{*}\right), b^{* *}\right\rangle \\
& =\left\langle a^{*} a, b^{* *}\right\rangle \\
& =\left\langle a, b^{* *} a^{*}\right\rangle,
\end{aligned}
$$

i.e., $\left(L_{A}\right)^{*}\left[u\left(a^{*}, b^{* *}\right)\right]=b^{* *} a^{*}$. Therefore,

$$
\begin{aligned}
\left\langle a^{*},\left(\Gamma \circ\left(L_{A}\right)^{* *}\right)\left(a^{* *}\right)\left(b^{* *}\right)\right\rangle & =\left\langle a^{*}, \Gamma\left(\left(L_{A}\right)^{* *}\left(a^{* *}\right)\right)\left(b^{* *}\right)\right\rangle \\
& =\left\langle u\left(a^{*}, b^{* *}\right),\left(L_{A}\right)^{* *}\left(a^{* *}\right)\right\rangle \\
& =\left\langle\left(L_{A}\right)^{*}\left[u\left(a^{*}, b^{* *}\right)\right], a^{* *}\right\rangle \\
& =\left\langle b^{* *} a^{*}, a^{* *}\right\rangle \\
& =\left\langle a^{*}, a^{* *} \square b^{* *}\right\rangle \\
& =\left\langle a^{*}, L_{\square}\left(a^{* *}\right)\left(b^{* *}\right)\right\rangle .
\end{aligned}
$$

The other identity follows analogously.

Lemma 2.2 Let $X, Y$ be complex normed spaces and let $T \in \mathfrak{B}(X, Y)$. Then $T^{*}$ is isometric if and only if $T\left([X]_{1}\right)^{-}=[Y]_{1}$, i.e., if and only if the closure of the image of the closed unit ball of $X$ under $T$ is the closed unit ball of $Y$. 
Proof If $T^{*}$ is isometric it has norm one. So, $\|T\|=1$ and $T\left([X]_{1}\right)^{-} \subseteq[Y]_{1}$. If this inclusión is strict let $y_{0} \in[Y]_{1}-T\left([X]_{1}\right)^{-}$. By the Hahn-Banach theorem, there exist a non-zero linear form $y_{1}^{*}$ on $Y$ and constants $r \in \mathbb{R}$ and $\delta>0$ so that

$$
\Re\left(\left\langle y, y_{1}^{*}\right\rangle\right)<r<r+\delta<\Re\left(\left\langle y_{0}, y_{1}^{*}\right\rangle\right)
$$

for all $y \in T\left([X]_{1}\right)^{-}$. It is easy to see that $y_{1}^{*}$ is bounded and with minor changes we can assume that $\left\|y_{1}^{*}\right\|=1$. It is also clear that $r>0$. Now, for $x \in[X]_{1}$ such that $\left\langle T(x), y_{1}^{*}\right\rangle \neq 0$ let $\varsigma \in \mathbb{C}$ so that $|\varsigma|=1$ and $\left\langle T(x), y_{1}^{*}\right\rangle=\varsigma\left|\left\langle T(x), y_{1}^{*}\right\rangle\right|$. Then

$$
\left|\left\langle x, T^{*}\left(y_{1}^{*}\right)\right\rangle\right|=\left\langle T(\bar{\zeta} x), y_{1}^{*}\right\rangle<r .
$$

By (2.1) we can conclude that $\left\|T^{*}\left(y_{1}^{*}\right)\right\|<\left\|y_{1}^{*}\right\|$, which contradicts the fact that $T^{*}$ is an isometry.

On the other hand, let us suppose that the condition holds. Hence it is plain that $\|T\|=1$ and so $\left\|T^{*}\right\|=1$. Further, given $\varepsilon>0$ and $y_{2}^{*} \in Y^{*}$ let $y \in[Y]_{1}$ so that $\left\|y_{2}^{*}\right\|-\varepsilon<\left|\left\langle y, y_{2}^{*}\right\rangle\right|$. By hypothesis and the continuity of $y_{2}^{*}$ we can choose $x_{1} \in[X]_{1}$ so that

$$
\left\|y_{2}^{*}\right\|-\varepsilon<\left|\left\langle T\left(x_{1}\right), y_{2}^{*}\right\rangle\right|=\mid\left\langle x_{1}, T^{*}\left(y_{2}^{*}\right)\right| \leq\left\|T^{*}\left(y_{2}^{*}\right)\right\| \leq\left\|y_{2}^{*}\right\|
$$

and the claim follows.

Theorem 2.3 The underlying algebra A inherits the same kind of stability of its second dual conjugate algebra.

Proof By Lemma 2.1 the map $\Gamma$ becomes isometric on the range of $\left(L_{A}\right)^{* *}$ (or $\left.\left(R_{A}\right)^{* *}\right)$ if $\left(A^{* *}, \square\right)$ (or $\left.\left(A^{* *}, \diamond\right)\right)$ is left (or right) stable. In these cases it is clear that $A$ becomes left (or right) stable.

If $a^{* *} \in A^{* *}$ we see that

$$
\begin{aligned}
\left\|R_{\square}\left(a^{* *}\right)\right\| & =\sup _{\left\|b^{* *}\right\|=1}\left\|b^{* *} \square a^{* *}\right\| \\
& =\sup _{\left\|b^{* *}\right\|=1\left\|a^{*}\right\|=1}\left|\left\langle a^{*}, b^{* *} \square a^{* *}\right\rangle\right| \\
& =\sup _{\left\|a^{*}\right\|=1\left\|b^{* *}\right\|=1}\left|\left\langle a^{* *} a^{*}, b^{* *}\right\rangle\right| \\
& =\sup _{\left\|a^{*}\right\|=1}\left\|a^{* *} a^{*}\right\| \\
& =\left\|\mathfrak{L}\left(a^{* *}\right)\right\|_{\mathfrak{B}\left(A^{*}\right)}
\end{aligned}
$$

where $\mathfrak{L}$ is the representation of $\left(A^{* *}, \square\right)$ into $\mathfrak{B}\left(A^{*}\right)$ so that for $a^{* *} \in A^{* *}$ and $a^{*} \in A^{*}$ acts as $\mathfrak{L}\left(a^{* *}\right)\left(a^{*}\right)=$ $a^{* *} a^{*}$. Consequently, $\left(A^{* *}, \square\right)$ is right stable if and only if $\mathfrak{L}$ is isometric. Moreover, let $b: \mathfrak{B}\left(A^{* *}\right) \rightarrow \mathfrak{B}\left(A^{*}\right)$ be the norm one projection $\Phi^{\mathrm{b}}=\left(\chi_{A}\right)^{*} \circ \Phi^{*} \circ \chi_{A^{*}}$ introduced in [6], p. 545. Following the notation of Lemma 2.1, for $a \in A, a^{*} \in A^{*}$ and $a^{* *} \in A^{* *}$ we have

$$
\begin{aligned}
\left\langle a,\left[b \circ \Gamma \circ\left(R_{A}\right)^{* *}\right]\left(a^{* *}\right)\left(a^{*}\right)\right\rangle & =\left\langle a,\left[\Gamma\left(\left(R_{A}\right)^{* *}\left(a^{* *}\right)\right)\right]^{\mathrm{b}}\left(a^{*}\right)\right\rangle \\
& =\left\langle\left[\Gamma\left(\left(R_{A}\right)^{* *}\left(a^{* *}\right)\right)\right]\left(\chi_{A}(a)\right), \chi_{A^{*}}\left(a^{*}\right)\right\rangle \\
& =\left\langle a^{*},\left[\Gamma\left(\left(R_{A}\right)^{* *}\left(a^{* *}\right)\right)\right]\left(\chi_{A}(a)\right)\right\rangle \\
& =\left\langle u\left(a^{*}, \chi_{A}(a)\right),\left(R_{A}\right)^{* *}\left(a^{* *}\right)\right\rangle \\
& =\left\langle\left(R_{A}\right)^{*}\left[u\left(a^{*}, \chi_{A}(a)\right)\right], a^{* *}\right\rangle \\
& =\left\langle a^{*} a, a^{* *}\right\rangle \\
& =\left\langle a, \mathfrak{L}\left(a^{* *}\right)\left(a^{*}\right)\right\rangle,
\end{aligned}
$$

i.e., $\mathfrak{L}=b \circ \Gamma \circ\left(R_{A}\right)^{* *}$. The maps $b, \Gamma$ and $\left(R_{A}\right)^{* *}$ are contractive. So, if $\mathfrak{L}$ is isometric then $\left(R_{A}\right)^{* *}$ must be isometric. But $\chi_{\mathfrak{B}(A)} \circ R_{A}=\left(R_{A}\right)^{* *} \circ \chi_{A}$ and as $\chi_{\mathfrak{B}(A)}$ and $\chi_{A}$ are isometric it is plain that $R_{A}$ becomes isometric.

Left stability of $A$ induced by left stability of $\left(A^{* *}, \diamond\right)$ follows analogously.

Example 2.4 Second conjugate algebras of stable Banach algebras could be not stable. For instance, let us consider the abelian Banach algebra $A=L^{1}[0,1]$ with respect to the usual convolution product. It is stable as it is provided by the Fejér kernel of a bounded approximate identity (cf. [7], 1.8.15). However, let $E \in L^{1}[0,1]^{* *}$ 
so that $\langle g, E\rangle=g(0)$ if $g \in C[0,1]$. Let $f \in L^{\infty}[0,1]$ and $g \in C[0,1]$. Then $f$ becomes absolutely integrable, $(f * g)(0)=0$ and $f * g \in C[0,1]$ because $C[0,1]$ is an ideal of $A$. So,

$$
\langle g, E f\rangle=\langle f * g, E\rangle=(f * g)(0)=0,
$$

i.e., $E f=0_{A^{*}}$ by the density of continuous functions within $A$. Now, given $F \in A^{* *}$ we obtain

$$
\left\langle f, R_{\square}(E)(F)\right\rangle=\langle f, F \square E\rangle=\langle E f, F\rangle=0 .
$$

We infer that $R_{\square}(E)=0_{\mathfrak{B}\left(A^{* *}\right)}$ and as evidently $E \neq 0_{A^{* *}}$ then $\left(A^{* *}, \square\right)$ is not right stable.

Theorem 2.5 Let $\zeta_{1}, \zeta_{2} \in \mathfrak{B}\left[A \hat{\otimes} A^{*}, A^{*}\right], \eta_{1}, \eta_{2} \in \mathfrak{B}\left[A^{*} \hat{\otimes} A^{* *}, A^{*}\right]$ be the unique operators so that

$$
\zeta_{1}\left(a \otimes a^{*}\right)=a^{*} a, \zeta_{2}\left(a \otimes a^{*}\right)=a a^{*}, \eta_{1}\left(a^{*} \otimes a^{* *}\right)=a^{* *} a^{*}, \eta_{2}\left(a^{*} \otimes a^{* *}\right)=a^{*} a^{* *},
$$

for all $a \in A, a^{*} \in A^{*}, a^{* *} \in A^{* *}$. Then:

(1) $\left(A^{* *}, \square\right)$ is RSBA if and only if $\zeta_{1}\left(\left[A \hat{\otimes} A^{*}\right]_{1}\right)^{-}=\left[A^{*}\right]_{1}$.

(2) $\left(A^{* *}, \square\right)$ is LSBA if and only if $\eta_{1}\left(\left[A^{*} \hat{\otimes} A^{* *}\right]_{1}\right)^{-}=\left[A^{*}\right]_{1}$.

(3) $\left(A^{* *}, \diamond\right)$ is RSBA if and only if $\eta_{2}\left(\left[A^{*} \hat{\otimes} A^{* *}\right]_{1}\right)^{-}=\left[A^{*}\right]_{1}$.

(4) $\left(A^{* *}, \diamond\right)$ is LSBA if and only if $\zeta_{2}\left(\left[A \hat{\otimes} A^{*}\right]_{1}\right)^{-}=\left[A^{*}\right]_{1}$.

Proof (1) It is worth mentioning the isometric isomorphism of Banach spaces $\left(A \hat{\otimes} A^{*}\right)^{*} \approx \mathfrak{B}\left(A^{*}\right)$. For, if $Q \in \mathfrak{B}\left(A^{*}\right)$ there is a unique $\theta_{Q} \in\left(A \hat{\otimes} A^{*}\right)^{*}$ so that $\left\langle a \otimes a^{*}, \theta_{Q}\right\rangle=\left\langle a, Q\left(a^{*}\right)\right\rangle$ on tensors. On the other hand, given $\theta \in\left(A \hat{\otimes} A^{*}\right)^{*}$ then $Q_{\theta} \in \mathfrak{B}\left(A^{*}\right)$ if we set $\left\langle a, Q_{\theta}\left(a^{*}\right)\right\rangle=\left\langle a \otimes a^{*}, \theta\right\rangle$. It is easy to see that the linear mappings $Q \rightarrow \theta_{Q}$ and $\theta \rightarrow Q_{\theta}$ are isometric and inverse of each other (cf. [5], Ch. VIII.2, Th. 1). In particular, $\zeta_{1}^{*} \in \mathfrak{B}\left[A^{* *},\left(A \hat{\otimes} A^{*}\right)^{*}\right]$ and given $a, a^{*}$ and $a^{* *}$ we have

$$
\begin{aligned}
\left\langle a, a^{* *} a^{*}\right\rangle & =\left\langle a^{*} a, a^{* *}\right\rangle \\
& =\left\langle\zeta_{1}\left(a \otimes a^{*}\right), a^{* *}\right\rangle \\
& =\left\langle a \otimes a^{*}, \zeta_{1}^{*}\left(a^{* *}\right)\right\rangle \\
& =\left\langle a, Q_{\zeta_{1}^{*}\left(a^{* *}\right)}\left(a^{*}\right)\right\rangle,
\end{aligned}
$$

i.e., $Q_{\zeta_{1}^{*}\left(a^{* *}\right)}\left(a^{*}\right)=a^{* *} a^{*}$ for all $a^{*}$ and $\left\|Q_{\zeta_{1}^{*}\left(a^{* *}\right)}\right\|=\left\|\zeta_{1}^{*}\left(a^{* *}\right)\right\|$. Moreover,

$$
\begin{aligned}
\left\|R_{\square}\left(a^{* *}\right)\right\| & =\sup _{\left\|b^{* *}\right\| \leq 1} \sup _{\left\|a^{*}\right\| \leq 1}\left|\left\langle a^{*}, b^{* *} \square a^{* *}\right\rangle\right| \\
& =\sup _{\left\|a^{*}\right\| \leq 1}\left\|a^{* *} a^{*}\right\| \\
& =\left\|\zeta_{1}^{*}\left(a^{* *}\right)\right\|
\end{aligned}
$$

and the assertion follows by Lemma 2.2.

(2) Following the notation of Lemma 2.1, by the universal property of projective tensor products there is a unique $u^{\wedge} \in \mathfrak{B}\left[A^{*} \hat{\otimes} A^{* *}, \mathfrak{B}(A)^{*}\right]$ so that $\|\hat{u}\|=\|u\|_{\mathfrak{B}\left[A^{*}, A^{* *} ; \mathfrak{B}(A)^{*}\right]}$ and for any $a^{*}$ and $a^{* *}$ the identity $u^{\wedge}\left(a^{*} \otimes a^{* *}\right)=u\left(a^{*}, a^{* *}\right)$ holds. If $n \in \mathfrak{B}(A)^{* *}$ we see that

$$
\begin{aligned}
\|\Gamma(n)\| & =\sup _{\left\|a^{* *}\right\| \leq 1}\left\|\Gamma(n)\left(a^{* *}\right)\right\| \\
& =\sup _{\left\|a^{* *}\right\| \leq 1} \sup _{\left\|a^{*}\right\|<1}\left|\left\langle u\left(a^{*}, a^{* *}\right), n\right\rangle\right| \\
& =\sup _{\left\|a^{* *}\right\| \leq 1} \sup _{\left\|a^{*}\right\| \leq 1}\left|\left\langle\hat{u}\left(a^{*} \otimes a^{* *}\right), n\right\rangle\right| \\
& =\sup _{\left\|a^{* *}\right\| \leq 1} \sup _{\left\|a^{*}\right\| \leq 1}\left|\left\langle\left(a^{*} \otimes a^{* *}\right),(\hat{u})^{*}(n)\right\rangle\right| .
\end{aligned}
$$

Hence, $\|\Gamma(n)\|=\left\|(\hat{u})^{*}(n)\right\|_{\left(A^{*} \hat{\otimes} A^{* *}\right)^{*}}$. Indeed, $\eta_{1}^{*}=(\hat{u})^{*} \circ\left(L_{A}\right)^{* *}$. Now, if $A^{* *}$ is a left stable Banach algebra by Lemma 2.1 the o-perators $\left(L_{A}\right)^{* *}$ and $\left.\Gamma\right|_{\Re\left[\left(L_{A}\right)^{* *}\right]}$ become both isometric. Hence, if $a^{* *} \in A^{* *}$ we have

$$
\left\|a^{* *}\right\|=\left\|\left(L_{A}\right)^{* *}\left(a^{* *}\right)\right\|
$$




$$
\begin{aligned}
& =\left\|\Gamma\left(\left(L_{A}\right)^{* *}\left(a^{* *}\right)\right)\right\| \\
& =\left\|(\hat{u})^{*}\left(\left(L_{A}\right)^{* *}\left(a^{* *}\right)\right)\right\| \\
& =\left\|\eta_{1}^{*}\left(a^{* *}\right)\right\|
\end{aligned}
$$

and $\eta_{1}^{*}$ becomes isometric. Therefore, by Lemma 2.2 we have

$$
\eta_{1}\left(\left[A^{*} \hat{\otimes} A^{* *}\right]_{1}\right)^{-}=\left[A^{*}\right]_{1}
$$

and the necessity follows.

Reciprocally, if $b^{* *} \in A^{* *}$ by Lemma 2.2 we can write

$$
\begin{aligned}
\left\|L_{\square}\left(b^{* *}\right)\right\| & =\left\|\Gamma\left[\left(L_{A}\right)^{* *}\left(b^{* *}\right)\right]\right\| \\
& =\left\|(\hat{u})^{*}\left[\left(L_{A}\right)^{* *}\left(b^{* *}\right)\right]\right\| \\
& =\left\|\eta_{1}^{*}\left(b^{* *}\right)\right\| \\
& =\left\|b^{* *}\right\| .
\end{aligned}
$$

(3) Here, we argue as in (2) since by Lemma 2.1 it is readily seen that $R_{\diamond}=\eta_{2}^{*}$.

(4) Similar argument as in (1).

Open Access This article is distributed under the terms of the Creative Commons Attribution 4.0 International License (http:// creativecommons.org/licenses/by/4.0/), which permits unrestricted use, distribution, and reproduction in any medium, provided you give appropriate credit to the original author(s) and the source, provide a link to the Creative Commons license, and indicate if changes were made.

\section{References}

1. Ansari-Piri, E.; Nouri, S.: Almost multipliers and some of their properties. J. Adv. Math. 11(7), 5397-5402 (2015)

2. Ansari-Piri, E.; Nouri, S.: Stable normed algebras. arXiv:1509.08142, 1-8 (2015)

3. Arens, R.: Operations induced in function classes. Monatsh. Math. 55, 1-19 (1951)

4. Arens, R.: The adjoint of a bilinear operation. Proc. Am. Math. Soc. 2, 839-848 (1951)

5. Diestel, J.; Uhl, J. J., Jr.: Vector measures. Am. Math. Surveys and Monogr. 15. AMS (1977)

6. Grosser, M.: Arens semi-regularity of the algebra of compact operators. Illinois J. Math. 31(4), 544-573 (1987)

7. Palmer, T.W.: Banach algebras and the general theory of *-algebras, vol. I. Cambridge University Press, Cambridge (2004)

8. Palmer, T.W.: The bidual of the compact operators. Trans. Am. Math. Soc. 288(2), 827-839 (1985)

Publisher's Note Springer Nature remains neutral with regard to jurisdictional claims in published maps and institutional affiliations. 